\title{
Antimalarial Activity of Fagaropsis angolensis (Rutaceae) Crude Extracts and Solvent Fractions of Its Stem Bark Against Plasmodium berghei in Mice
}

This article was published in the following Dove Press journal: Journal of Experimental Pharmacology

\section{Belete Kassa Alemu (D) Desye Misganaw (D)}

Pharmacology and Toxicology Unit, Department of Pharmacy, College of Medicine and Health Sciences, Wollo University, Dessie, Ethiopia
Correspondence: Belete Kassa Alemu Pharmacology and Toxicology Unit, Department of Pharmacy, College of Medicine and Health Sciences, Wollo University, P O Box I I45, Dessie, Ethiopia

Tel +25 I 918330307

$\mathrm{Fax}+251333115052$

Email belete.kassa@wu.edu.et
Background: A current challenge in malaria control and elimination is the progressive resistance to most antimalarial drugs which necessitates the discovery of new options. Hence, the current study was initiated to investigate the antimalarial activity of the stem bark of Fagaropsis angolensis in mice.

Methods: The test material was extracted using $80 \%$ methanol in a cold maceration technique and further fractionated in solvents of varied polarity. Acute oral toxicity was assessed following the OECD guideline no. 425 protocol. Then, the antimalarial activities of the crude extract and the fractions were evaluated in a 4-day suppression test. Rane's test was also used to evaluate the curative potential of the n-butanol fraction that showed the highest effect during the 4-day suppressive test. Parameters such as parasitemia suppression, mean survival time, packed cell volume, rectal temperature, and body weight were determined to establish the activity.

Results: The acute oral toxicity test indicated that the plant did not cause any signs of behavioral changes or mortality at $200 \mathrm{mg} / \mathrm{kg}$ limit dose. In a 4-day suppression test, a significant dose-dependent reduction in the parasitemia level and prolongation of survival time were observed $(\mathrm{p}<0.001)$ in all three doses of the crude extract compared with the negative control. The crude extract also exhibited a significant $(\mathrm{p}<0.001)$ protective effect in packed cell volume and rectal temperature decline in all three doses in a dose-dependent fashion compared with the negative control. Among all fractions, the n-butanol fraction displayed the highest effects in all parameters in the 4-day suppression test. In addition, the n-butanol fraction also showed a significant percentage of parasitemia suppression effects at all doses in Rane's test. Furthermore, higher free radical scavenging activity was observed in the n-butanol fraction and the $80 \%$ methanol extract.

Conclusion: This study established that Fagaropsis angolensis had shown potential antimalarial activity as evidenced by the significant effects in the different parameters, upholding its traditional use for the treatment of malaria and laying the foundation for further investigations. Keywords: antimalarial activity, Plasmodium berghei, Fagaropsis angolensis, parasitemia, antioxidant activity

\section{Background}

Malaria, a serious worldwide protozoan disease, is a common killer predominantly in tropical and subtropical countries, primarily affecting children and pregnant women. There are five plasmodium species known to cause malaria infection to date, of which Plasmodium falciparum and Plasmodium vivax lead to most malaria infections globally. ${ }^{1-3}$ 
The 5 years trend reports of malaria cases by the World Health Organization (WHO) showed that $21 \%$ incidence and $29 \%$ mortality rates of malaria occur worldwide. In 2018, more than 228 million cases and more than 400,000 deaths occurred due to malaria, of which about 213 million cases and 380 thousand deaths occurred in Africa. ${ }^{3,4}$ SubSaharan Africa, in particular, is a high burden region where more than $90 \%$ of the global malaria-related mortality occurs. ${ }^{5}$

Currently, the major challenge in malaria control and elimination is that most antimalarial drugs (chloroquine, artemether/lumefantrine, quinine, primaquine, etc.) have reduced efficacy and fail to treat malaria due to the progressive resistance of $P$. falciparum and $P$. vivax. ${ }^{6}$ In addition to this, no single antimalarial drug is effective against all exo-erythrocytic and intra-erythrocytic forms of the parasite. ${ }^{6,7}$ Moreover, the elimination of malaria and control of transmission is hindered by the lack of sporozoiticidal drugs. The increasing resistance of the parasites to the currently available antimalarial drugs and their limited therapeutic efficacy alongside the slow pace in the discovery and development of new alternative drugs make the situation more challenging. Hence the necessity in searching for any new treatment options, primarily from medicinal plants. ${ }^{8,9}$ Historically, medicinal plants have long been a potential source of many drugs including the available antimalarial drugs. ${ }^{10}$

The studied plant, Fagaropsis angolensis, is a tree that belongs to the Rutaceae family of flowering plants. The different parts of the plant are traditionally used for the treatment of various ailments. For instance, leaves and root decoctions are used to treat back pain and joint-aches, malaria, male sterility, and cancer. ${ }^{11-13}$ The seeds of the plant are also chewed for malaria. ${ }^{14}$ In Ethiopia and Kenya, a decoction of the stem bark is widely used in the treatment of malaria, pneumonia, amoebiasis, and diarrhea. ${ }^{15,16}$ Traditionally, the plant is also applied in veterinary uses to treat diarrhea, wounds, bovine pleuropneumonia, babesiosis, and anaplasmosis in cattle. ${ }^{17-19}$

Moreover, previous reports in literature indicated that $F$. angolensis possessed antimicrobial, anticancer, anti-leishmanial, antifungal, and anti-trypanosomal activities. ${ }^{20-24}$ Most importantly, some studies revealed that the aqueous and methanolic stem bark extracts of $F$. angolensis showed significant in vitro anti-plasmodial activity against both chloroquine-resistant and chloroquine-sensitive strains of $P$. falciparum. ${ }^{25-27}$ Therefore, the present study was initiated to investigate the antimalarial activity of the stem bark extract of $F$. angolensis in Plasmodium bergheiinfected mice and its antioxidant activity.

\section{Methods and Materials Plant Material}

The stem bark of $F$. angolensis was collected from Sidama, Southern Ethiopia in December 2019. The plant material was identified and authenticated and a specimen number (BK003) was deposited at the National Herbarium, College of Natural and Computational Sciences, University of Gondar for future reference.

\section{Experimental Animals and Parasite}

Healthy adult male Swiss albino mice (25-30 g, and 6-8 weeks of age) (for the antimalarial test) and female mice (for the acute toxicity test) were obtained from the animal house of the Department of Pharmacy, Wollo University. The animals were placed in cages under standard conditions with 12 hours light and dark cycles. They were provided with a standard pellet diet and water ad libitum throughout the experiment and acclimatized to laboratory conditions for one week before the experiment. Chloroquine-sensitive $P$. berghei ANKA strain was obtained from the Ethiopian Health and Nutrition Research Institute (EHNRI). The parasite was maintained by serial passage of blood from infected mice to noninfected mice on a weekly basis. ${ }^{28}$

\section{Extraction and Solvent Fractionation}

After the stem bark of the plant was dried for 3 weeks in the shade, it was ground to a coarse powder using a wooden mortar and pestle. The coarse powder was weighed $(600 \mathrm{~g})$ and macerated in Erlenmeyer flasks with $80 \%$ methanol $(1.8 \mathrm{~L})$ for 72 hours with occasional shaking at room temperature. Then, the extract was filtered by gauze (muslin) and re-filtered using Whatman No. 1 filter paper. The bark was re-macerated twice in the same manner. Then, the filtrates were combined and evaporated on a rotary evaporator (Buchi Rota Vapor R-200) under reduced pressure and dried in a lyophilizer (Wagtech Jouan Nordic DK-3450 Allerod, Denmark). The extract was put in the refrigerator until used for further activities.

Then, the hydromethanolic extract was successively fractionated using solvents of differing polarity (chloroform, n-butanol, and distilled water). The crude extract $(60 \mathrm{~g})$ was mixed with $200 \mathrm{~mL}$ distilled water in a separating funnel and an equivalent amount of chloroform was 
added and shaken. After a distinct layer was formed in the mixture, the chloroform fraction was separated. This procedure was repeated three times to find the chloroform fraction. Then the aqueous residue was mixed with an equivalent volume of n-butanol and separated. The chloroform and n-butanol fractions were evaporated under reduced pressure using a rotary evaporator (Buchi Rota Vapor R-200) and dried in an oven at $40^{\circ} \mathrm{C}$, while the aqueous fraction was dried in a freeze drier. The dried fractions were placed in a airtight container at $-4^{\circ} \mathrm{C}$ until used for the actual experiment.

\section{Acute Oral Toxicity Study}

Acute oral toxicity study was carried out based on the Organization for Economic Cooperation and Development (OECD) guidelines no. $425 .{ }^{29}$ Initially, the $80 \%$ methanol extract and solvent fractions were administered to mice at a dose of $2000 \mathrm{mg} / \mathrm{kg}$, after fasting for 4 hours. Then, a mouse was kept under strict observation of physical and behavioral changes for $24 \mathrm{~h}$, with special attention during the first $4 \mathrm{~h}$. Following this pilot study, another four mice were allowed to fast for 4 hours having obtained the information about safety in the initial study. The same $80 \%$ methanol extract and solvent fractions were administered separately to each mouse and were observed for $24 \mathrm{~h}$. The animals were also observed for the development of any toxicity daily for 14 days.

\section{Animal Grouping and Dosing}

The mice were divided into five groups randomly, each group containing six mice $(\mathrm{n}=6)$. Group I (negative control) was treated with $10 \mathrm{~mL} / \mathrm{kg}$ distilled water (DW); Groups II, III, and IV were treated with three different doses (200, 400, and $600 \mathrm{mg} / \mathrm{kg}$, respectively) and Group $\mathrm{V}$ was treated with the standard drug, chloroquine $(25 \mathrm{mg}$ / $\mathrm{kg}$ ) in both 4-day suppressive and curative model. ${ }^{30}$

\section{Parasite Inoculation}

Previously infected mice having a parasitemia level of 20 $30 \%$ were taken as donors. Donor mice were then sacrificed by cervical dislocation and blood was collected by a heparinized tube containing $0.5 \%$ tri-sodium citrate. Then the blood was diluted with normal saline $(0.9 \%)$ based on the parasitemia level of the donor mice and the red blood cell (RBC) count of normal mice on the basis that $1 \mathrm{~mL}$ blood contains $5 \times 10^{7}$ infected RBCs. Then, each mouse was inoculated with $0.2 \mathrm{~mL}$ of blood containing $1 \times 10^{7} \mathrm{P}$. berghei parasitized erythrocytes by intraperitoneal route. ${ }^{31-33}$

\section{Evaluation of Antimalarial Activity Four-Day Suppressive Test (Peter's Test)}

In this experiment, 30 mice were infected on day 0 . Then, 2 hours post-infection, mice were distributed randomly and treated according to their grouping as described above. Treatment of the $80 \%$ methanol extract and the fractions continued until day 3. Thereafter, on day 4 , determination of parasitemia level, percent suppression, and survival time were undertaken and additional parameters such as packed cell volume (PCV), body weight, and temperature were measured before and at the end of the experiment. ${ }^{31,34}$

\section{Curative Test (Rane's Test)}

The most active fraction during the 4-day suppressive test was evaluated for its curative potential through Rane's test. At 72 hours post-infection, mice were grouped randomly into five groups and treated accordingly as above. Mice were further treated for 3 consecutive days. The daily parasitemia level was recorded until the 7th day. Similarly, PCV, body weight, and temperature were measured before and at the end of the experiment. ${ }^{35}$

\section{Determination of Parasitemia and Survival Time}

Determination of parasitemia was carried out by counting the number of infected RBCs (a minimum of three fields per slide) using a light microscope (MB23 $0 \mathrm{~T}$, China) with a lens magnification power of 100x. Percent parasitemia and percent suppression were calculated using the following formula. ${ }^{36}$

$$
\begin{aligned}
\% \text { Parasitemia } & =\frac{\text { Number of parasitized } R B C}{\text { Total number of } R B C \text { count }} \times 100 \\
\% \text { Suppression }= & \frac{\begin{array}{l}
\% \text { Parasitemia in } \\
\text { negative control }-\begin{array}{l}
\% \text { Parasitemia in } \\
\text { study group }
\end{array}
\end{array}}{\begin{array}{l}
\text { \%parasitemia in } \\
\text { negative control }
\end{array}} \\
& \times 100 \quad \text { a }
\end{aligned}
$$

Finally, the mean survival time (MST) was computed by using the formula. ${ }^{31,34}$

$$
\text { MST }=\frac{\text { Total number of days mice survived }}{\text { Total number of mice }}
$$

Determination of Packed Cell Volume, Rectal Temperature, and Body Weight

PCV determination was conducted after blood was collected from the tail of each mouse using heparinized capillary tubes. A microhematocrit centrifuge 
(Hettichhaematokrit, Germany), centrifugation at 12,000 rpm for $5 \mathrm{~min}$, was used. In addition, sensitive digital weighing balance and rectal thermometer were used to measure body weight and rectal temperature of each mouse, respectively. ${ }^{37}$

$$
P V C=\frac{\text { Volume of erythrocytes in a given volume of blood }}{\text { Total blood volume }}
$$

\section{Preliminary Phytochemical Analysis}

The phytochemical analysis of $80 \%$ methanol extract and fractions were performed following the methods described by Trease and Evans, and Debella. ${ }^{38,39}$

\section{In vitro Antioxidant Activity in DPPH Assay}

The free radical scavenging activities of the crude extract and solvent fractions were determined by using diphenyl2-picrylhydrazyl (DPPH; Sigma Aldrich) assays following the methods described by Blois ${ }^{40}$ and Desmarchelier et al. ${ }^{41}$ Different concentrations of $100 \mathrm{~mL}$ of a methanolic solution ranging from 50 to $1000 \mathrm{mg} / \mathrm{mL}$ were added to $3.9 \mathrm{~mL}$ of a $0.004 \%$ methanolic solution of DPPH. The absorbance was measured at $517 \mathrm{~nm}$ after $30 \mathrm{~min}$, and the percent inhibition of DPPH was calculated. The percentage of the scavenging of the DPPH free radical was calculated by the formula: $(\mathrm{A} 0-\mathrm{A} 1) / \mathrm{A} 0 * 100$, where $\mathrm{A} 0$ is the absorbance of the control and A1 is the absorbance of the extract/standard.

\section{Data Processing and Analysis}

The data were entered, coded, and analyzed using Statistical Package for the Social Sciences (SPSS) version 23 and presented as mean \pm standard error of the mean (SEM). Oneway analysis of variance (ANOVA) followed by Tukey post hoc test was performed. Furthermore, two-way repeated measures ANOVA was employed for the analysis of the gradual development of parasitemia across days of treatment in Rane's test. Finally, the results were considered significant at a $95 \%$ confidence level when the $p$-value was $<0.05$.

\section{Results}

\section{Acute Oral Toxicity Test}

Oral administration of $2000 \mathrm{mg} / \mathrm{kg}$ of $80 \%$ methanol extract and the fractions produced no signs of behavioral changes within $24 \mathrm{~h}$ of application. The animals were also followed up for 14 days and neither mortality nor any signs of toxicity were observed, indicating that the lethal dose (LD50) values of the extract and fractions are exceeding $2000 \mathrm{mg} / \mathrm{kg}$ in mice.

\section{Effect of $80 \%$ Methanol Extract in the 4- Day Suppressive Test}

A dose-dependent reduction in the parasitemia level and prolonged MST were significantly $(\mathrm{p}<0.001)$ different in the three doses of the methanol extract compared with the negative controls. The highest dose $(600 \mathrm{mg} / \mathrm{kg})$ was found to produce a significant reduction $(\mathrm{p}<0.001)$ in parasitemia and an increased survival time compared with 200 and $400 \mathrm{mg} / \mathrm{kg}$ doses. However, all the three doses showed significantly lower effects than the standard drug $(\mathrm{p}<0.001)$ (Table 1).

With respect to $\mathrm{PCV}$ and temperature determination, the crude extract exhibited a significant $(\mathrm{p}<0.001)$ protective effect in PCV and rectal temperature decline in all three doses in a dose-dependent fashion when compared with the negative control. The highest dose was found to have a statistically significant effect in comparison with the middle $(\mathrm{p}<0.05)$ and the lowest dose $(\mathrm{p}<0.01)$ in these parameters. In addition, the crude extract was able to prevent bodyweight reduction significantly $(\mathrm{p}<0.01)$ compared with the negative control at the doses of $400 \mathrm{mg} / \mathrm{kg}$ and $600 \mathrm{mg} / \mathrm{kg}$. However, significantly $(\mathrm{p}<0.001)$ more protective effects were produced by the standard drug than all the doses of the crude extract towards a reduction in the three parameters (Table 2).

\section{Effect of Solvent Fractions in the 4-Day Suppressive Test}

Despite all the fractions having varying degrees of parasitemia suppression and survival prolongation effects, every dose of the fractions showed a significant effect $(p<0.001)$ in parasitemia suppression as well as an increase in survival

Table I Parasitemia and Survival Time of Infected Mice Treated with $80 \%$ Methanol Extract of the Stem Bark of F. angolensis in the 4-Day Suppressive Test

\begin{tabular}{|c|c|c|c|}
\hline Groups & \% Parasitemia & \% Suppression & $\begin{array}{l}\text { Survival Time } \\
\text { (Days) }\end{array}$ \\
\hline $2 \%$ TW80 & $37.48 \pm 0.25$ & - & $7.87 \pm 0.25$ \\
\hline $\mathrm{CQ} 25 \mathrm{mg} / \mathrm{kg}$ & $0.00 \pm 0.00$ & $100.00^{\mathrm{a}^{* * * k}}$ & $28.65 \pm 0.00^{\mathrm{a}^{* *+* k}}$ \\
\hline 200 mg/kg & $18.72 \pm 0.45$ & 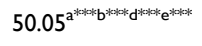 & 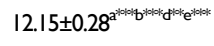 \\
\hline $400 \mathrm{mg} / \mathrm{kg}$ & $16.94 \pm 0.16$ & $54.80^{\mathrm{a}^{* * * * \mathrm{~b}} \mathrm{~b}^{* * *} \mathrm{e} \mathrm{e}^{* * *}}$ & 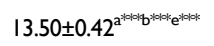 \\
\hline $600 \mathrm{mg} / \mathrm{kg}$ & $15.12 \pm 0.31$ & 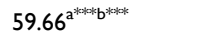 & 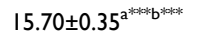 \\
\hline
\end{tabular}

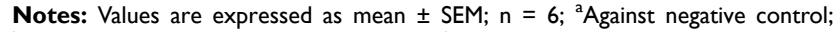
${ }^{b}$ Against CQ25 mg/kg; 'Against $200 \mathrm{mg} / \mathrm{kg}$; ${ }^{\mathrm{d} A g a i n s t} 400 \mathrm{mg} / \mathrm{kg}$; ${ }^{\mathrm{e}}$ Against $600 \mathrm{mg} / \mathrm{kg}$; $* * p<0.05 ; * * 0<0.01 ; * * *^{3} p<0.001$.

Abbreviations: $2 \%$ TW80, $2 \%$ Tween80; CQ, chloroquine. 
Table 2 Packed Cell Volume, Rectal Temperature and Body Weight of Infected Mice Treated with $80 \%$ Methanol Extract of the Stem Bark of F. angolensis In the 4-Day Suppressive Test

\begin{tabular}{|c|c|c|c|c|c|c|c|c|c|}
\hline \multirow[t]{2}{*}{ Groups } & \multicolumn{3}{|c|}{ Packed Cell Volume } & \multicolumn{3}{|c|}{ Rectal Temperature } & \multicolumn{3}{|c|}{ Body Weight } \\
\hline & $\mathbf{D}_{0}$ & $\mathbf{D}_{4}$ & $\%$ Change & $\mathbf{D}_{0}$ & $\mathbf{D}_{4}$ & $\%$ Change & $\mathbf{D}_{0}$ & $\mathbf{D}_{4}$ & $\%$ Change \\
\hline 2\% TW80 & $60.82 \pm 1.17$ & $57.36 \pm 1.02$ & -6.03 & $36.86 \pm 0.16$ & $34.73 \pm 0.16$ & -6.13 & $28.27 \pm 0.21$ & $26.85 \pm 0.22$ & -5.29 \\
\hline CQ25 mg/kg & $60.76 \pm 1.33$ & $60.53 \pm 1.14$ & $-0.38^{\mathrm{a}^{* * * *}}$ & $36.54 \pm 0.25$ & $36.23 \pm 0.39$ & $-0.86^{\mathrm{a}^{* * *}}$ & $28.84 \pm 0.32$ & $28.34 \pm 0.29$ & $-1.76^{\mathrm{a}^{* * * *}}$ \\
\hline 200 mg/kg & $60.55 \pm 0.57$ & $57.96 \pm 0.48$ & $-4.47^{\mathrm{a} * * * \mathrm{~b}} \mathrm{~b}^{* * * *} \mathrm{e}^{* * *}$ & $36.52 \pm 0.13$ & $35.48 \pm 0.18$ & $-2.93^{\mathrm{a}^{* * * *} \mathrm{~b}^{* * * *} \mathrm{e}^{*}}$ & $28.75 \pm 0.32$ & $27.75 \pm 0.34$ & $-3.60^{\mathrm{b} * * *}$ \\
\hline $400 \mathrm{mg} / \mathrm{kg}$ & $60.80 \pm 0.69$ & $58.33 \pm 0.54$ & $-3.45^{\mathrm{a}^{* * * *} \mathrm{~b}^{* * * *} \mathrm{e}^{*}}$ & $36.40 \pm 0.22$ & $35.52 \pm 0.19$ & $-2.48^{\mathrm{a}^{* * * * *} \mathrm{~b}^{* * * *}}$ & $28.81 \pm 0.44$ & $27.96 \pm 0.35$ & $-3.04^{\mathrm{a}^{* * *} \mathrm{~b} * * * *}$ \\
\hline $600 \mathrm{mg} / \mathrm{kg}$ & $60.91 \pm 0.45$ & $59.99 \pm 0.52$ & $-1.53^{\mathrm{a}^{* * * *} \mathrm{~b} * * *}$ & $36.25 \pm 0.18$ & $35.62 \pm 0.20$ & $-1.77^{\mathrm{a}^{* * * *} \mathrm{~b} * * *}$ & $28.42 \pm 0.54$ & $27.64 \pm 0.66$ & $-2.82^{\mathrm{a}^{* *} \mathrm{~b} * * *}$ \\
\hline
\end{tabular}

Notes: Values are expressed as mean \pm SEM; $\mathrm{n}=6{ }^{2}{ }^{\mathrm{a}} \mathrm{Against}$ negative control; ${ }^{\mathrm{b}}$ Against CQ25 mg/kg; ${ }^{\mathrm{C}} \mathrm{Against} 200 \mathrm{mg} / \mathrm{kg} ;{ }^{\mathrm{d}} \mathrm{Against} 400 \mathrm{mg} / \mathrm{kg} ;{ }^{\mathrm{e}} \mathrm{Against} 600 \mathrm{mg} / \mathrm{kg} ;{ }^{*} \mathrm{p}<0.05$; $* * 0<0.01 ; * * * p<0.001$.

Abbreviations: $2 \%$ TW80, $2 \%$ Tween80; CQ, chloroquine; $D_{0}$, pre-treatment value on day 0 ; $D_{4}$, post-treatment value on day 4 .

time compared with their respective negative controls. The highest doses of the n-butanol and chloroform fractions had a significant parasitemia suppression effect $(\mathrm{p}<0.01)$ in comparison with their respective lowest doses with a p-value of $\mathrm{p}<0.01$ and $\mathrm{p}<0.05$, respectively. Likewise, a dose-dependent effect was also observed in the two fractions. Nonetheless, the n-butanol fraction showed the highest effects among the fractions in parasitemia suppression effect as well as prolongation of survival time (Table 3).

All the doses of the three fractions produced a statistically significant protective effect $(\mathrm{p}<0.001)$ in PCV decline when compared with the negative controls. However, the n-butanol fraction indicated a higher significant preventive effect $(p<0.01)$ in rectal temperature decline than the chloroform and aqueous fractions, compared with their negative controls.
In addition, the highest doses were able to show a dose-dependent temperature stabilization effect in comparison with the middle and lowest doses, in all three fractions. Regarding body weight change, the n-butanol and chloroform fractions showed significant prevention of body weight lowering at 400 and 600 $\mathrm{mg} / \mathrm{kg}$ doses $(\mathrm{p}<0.01)$ and at $200 \mathrm{mg} / \mathrm{kg}(\mathrm{P}<0.05)$, compared with the negative controls. But, the aqueous fraction was devoid of a protective effect at all doses (Table 4).

\section{Effect of $n$-Butanol Fraction in the Rane's Test}

Among all fractions, the n-butanol fraction displayed the highest effects in all parameters in the 4-day suppression test model. As a result, it was further investigated for its curative potential in Rane's test. In this experiment, all three doses of

Table 3 Parasitemia and Survival Time of Infected Mice Treated with Solvent Fractions of the Stem Bark of F. angolensis in the 4-Day Suppressive Test

\begin{tabular}{|c|c|c|c|}
\hline Groups & \% Parasitemia & \% Suppression & Survival Time (Days) \\
\hline $2 \%$ TW80 & $37.78 \pm 0.64$ & - & $7.45 \pm 0.22$ \\
\hline $\mathrm{CQ} 25 \mathrm{mg} / \mathrm{kg}$ & $0.00 \pm 0.00$ & $100.00^{\mathrm{a}^{* * * *}}$ & $28.00 \pm 0.00^{\mathrm{a}^{* * * *}}$ \\
\hline $200 \mathrm{mg} / \mathrm{kg} \mathrm{CF}$ & $25.62 \pm 0.44$ & $32.19^{\mathrm{a}^{* * *} \mathrm{~b} \mathrm{~b}^{* * * *} \mathrm{~d} * * * \mathrm{e}^{* *}}$ & $10.15 \pm 0.25^{\mathrm{a}^{* * * *} \mathrm{~b}^{* * * *} \mathrm{e}^{*}}$ \\
\hline $400 \mathrm{mg} / \mathrm{kg} \mathrm{CF}$ & $23.76 \pm 0.35$ & $37.11^{\mathrm{a}^{* * * \mathrm{~b}^{* * * * *}}}$ & $11.33 \pm 0.39^{\mathrm{a}^{* * * *} \mathrm{~b}^{* * * *}}$ \\
\hline $600 \mathrm{mg} / \mathrm{kg} \mathrm{CF}$ & $22.48 \pm 0.21$ & $40.50^{\mathrm{a} * * * \mathrm{~b} * * *}$ & $12.02 \pm 0.27^{\mathrm{a} * * * \mathrm{~b} * * *}$ \\
\hline $2 \%$ TW 80 & $37.92 \pm 0.65$ & - & $7.18 \pm .017$ \\
\hline $\mathrm{CQ} 25 \mathrm{mg} / \mathrm{kg}$ & $0.00 \pm 0.00$ & $100.00^{\mathrm{a} * * *}$ & $28.00 \pm 0.00^{\mathrm{a}^{* * * *}}$ \\
\hline $200 \mathrm{mg} / \mathrm{kg} \mathrm{BF}$ & $21.35 \pm 0.38$ & 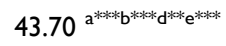 & $11.85 \pm 0.39^{\mathrm{a}^{* * *} \mathrm{~b}^{* * * *} \mathrm{~d} * \mathrm{e}^{*}}$ \\
\hline $400 \mathrm{mg} / \mathrm{kg} \mathrm{BF}$ & $19.66 \pm 0.34$ & $48.15^{\mathrm{a}^{* * * \mathrm{~b}^{* * * * *}}}$ & $12.88 \pm 0.23^{\mathrm{a}^{* * * * \mathrm{~b} * * * *} \mathrm{e}^{*}}$ \\
\hline $600 \mathrm{mg} / \mathrm{kg} \mathrm{BF}$ & $17.85 \pm 0.36$ & $52.93^{\mathrm{a} * * * \mathrm{~b} * * *}$ & $\mid 4.78 \pm 0.26^{\mathrm{a} * * * b^{* * * *}}$ \\
\hline $2 \%$ TW 80 & $37.85 \pm 0.42$ & - & $7.17 \pm 0.23$ \\
\hline CQ25 mg/kg & $0.00 \pm 0.00$ & $100.00^{\mathrm{a}^{* * * *}}$ & $28.00 \pm 0.00^{\mathrm{a}^{* * * *}}$ \\
\hline $200 \mathrm{mg} / \mathrm{kg} \mathrm{AF}$ & $33.56 \pm 0.38$ & $11.33^{\mathrm{a}^{* * * * * * * * *} \mathrm{e}^{*}}$ & 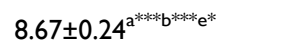 \\
\hline $400 \mathrm{mg} / \mathrm{kg} \mathrm{AF}$ & $31.44 \pm 0.27$ & $16.94^{\mathrm{a}^{* * * * \mathrm{~b} * * * *}}$ & $9.14 \pm 0.26^{\mathrm{a} * * * \mathrm{~b} * * * *}$ \\
\hline $600 \mathrm{mg} / \mathrm{kg} \mathrm{AF}$ & $30.45 \pm 0.23$ & 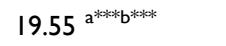 & $9.45 \pm 0.22^{\mathrm{a}^{* * * *} \mathrm{~b}^{* * * *}}$ \\
\hline
\end{tabular}

Notes: Values are expressed as mean \pm SEM; $\mathrm{n}=6$; ${ }^{\mathrm{a}}$ Against negative control; ${ }^{\mathrm{b}}$ Against CQ25 mg/kg; ${ }^{\mathrm{C}}$ Against $200 \mathrm{mg} / \mathrm{kg}$; ${ }^{\mathrm{d}} \mathrm{Against} 400 \mathrm{mg} / \mathrm{kg} ;{ }^{\mathrm{e}} \mathrm{Against} 600 \mathrm{mg} / \mathrm{kg} ;{ }^{*} \mathrm{p}<0.05$; $* * \mathrm{p}<0.01$; *** $\mathrm{p}<0.001$.

Abbreviations: $\mathrm{CF}$, chloroform fraction; $\mathrm{BF}$, butanol fraction; $\mathrm{AF}$, aqueous fraction; $2 \% \mathrm{TW} 80,2 \%$ Tween 80 ; CQ, chloroquine. 
Table 4 Packed Cell Volume, Rectal Temperature and Body Weight of Infected Mice Treated with Solvent Fractions of the Stem Bark of $F$. angolensis in the 4-Day Suppressive Test

\begin{tabular}{|c|c|c|c|c|c|c|c|c|c|}
\hline \multirow[t]{2}{*}{ Groups } & \multicolumn{3}{|c|}{ Packed Cell Volume } & \multicolumn{3}{|c|}{ Rectal Temperature } & \multicolumn{3}{|c|}{ Body Weight } \\
\hline & $D_{0}$ & $\mathbf{D}_{4}$ & $\%$ Change & $\mathbf{D}_{0}$ & $\mathbf{D}_{4}$ & $\%$ Change & $D_{0}$ & $\mathbf{D}_{4}$ & $\%$ Change \\
\hline $2 \%$ TW80 & $59.34 \pm 0.14$ & $54.05 \pm 0.42$ & -9.79 & $36.52 \pm 0.14$ & $34.62 \pm 0.33$ & -5.49 & $28.382 \pm 0.21$ & $27.070 \pm 0.19$ & -4.85 \\
\hline $\mathrm{CQ} 25 \mathrm{mg} / \mathrm{kg}$ & $60.15 \pm 0.31$ & $59.82 \pm 0.24$ & $-0.55^{\mathrm{a}^{* * * *}}$ & $35.48 \pm 0.04$ & $35.50 \pm 0.16$ & $-0.06^{\mathrm{a} * * *}$ & $28.082 \pm 0.44$ & $28.42 \pm 0.22$ & $-1.19^{\mathrm{a}^{* * * *}}$ \\
\hline $200 \mathrm{mg} / \mathrm{kg} \mathrm{CF}$ & $59.53 \pm 0.13$ & $55.91 \pm 0.55$ & $-6.47^{\mathrm{a}^{* * * * *} \mathrm{~b} * * * *}$ & $36.31 \pm 0.11$ & $34.96 \pm 0.24$ & $-3.86^{\mathrm{a}^{*} \mathrm{~b}^{* * * *}}$ & $28.142 \pm 0.09$ & $26.96 \pm 0.35$ & $-4.38^{\mathrm{a}^{*} \mathrm{~b}^{* * * * *}}$ \\
\hline $400 \mathrm{mg} / \mathrm{kg} \mathrm{CF}$ & $60.01 \pm 0.33$ & $56.80 \pm 0.46$ & $-5.65^{a^{* * *}+b^{* * * * *}}$ & $36.20 \pm 0.08$ & $35.44 \pm 0.64$ & $-2.14^{\mathrm{a} * * \mathrm{~b} * * *}$ & $27.972 \pm 0.55$ & $26.98 \pm 0.42$ & $-3.68^{\mathrm{a}^{*} \mathrm{~b} * * *}$ \\
\hline $600 \mathrm{mg} / \mathrm{kg} \mathrm{CF}$ & $60.71 \pm 0.23$ & $57.83 \pm 0.36$ & $-4.798^{\mathrm{a} * * * * b^{* * * * *}}$ & $35.73 \pm 0.34$ & $35.01 \pm 0.78$ & $-2.06^{\mathrm{a} * \mathrm{~b}^{*} \mathrm{b**}}$ & $28.162 \pm 0.33$ & $27.21 \pm 0.42$ & $-3.50^{\mathrm{a} * * \mathrm{~b} * * *}$ \\
\hline $2 \%$ TW80 & $59.24 \pm 0.11$ & $54.04 \pm 0.32$ & -9.62 & $36.53 \pm 0.14$ & $34.59 \pm 0.71$ & -5.61 & $28.40 \pm 0.19$ & $27.07 \pm 0.22$ & -4.91 \\
\hline $\mathrm{CQ} 25 \mathrm{mg} / \mathrm{kg}$ & $60.14 \pm 0.09$ & $59.95 \pm 0.18$ & $-0.32^{\mathrm{a}^{* * * *}}$ & $35.49 \pm 0.44$ & $35.49 \pm 0.65$ & $-0.00^{\mathrm{a}^{* * * *}}$ & $28.10 \pm 0.36$ & $28.32 \pm 0.21$ & $-0.78^{\mathrm{a}^{* * *}}$ \\
\hline $200 \mathrm{mg} / \mathrm{kg} \mathrm{BF}$ & $59.48 \pm 0.23$ & $56.82 \pm 0.24$ & $-4.68^{\mathrm{a} * * * \mathrm{~b} * * * *} \mathrm{~d}^{*} \mathrm{e}^{* * *}$ & $36.37 \pm 0.34$ & $35.07 \pm 0.09$ & $-3.7 I^{\mathrm{a}^{*} \mathrm{~b}^{* * * * *} \mathrm{e}^{* * *}}$ & $28.01 \pm 0.39$ & $26.95 \pm 0.19$ & $-3.93^{\mathrm{a}^{*} \mathrm{~b}^{* * * *}}$ \\
\hline $400 \mathrm{mg} / \mathrm{kg} \mathrm{BF}$ & $60.10 \pm 0.53$ & $57.86 \pm 0.50$ & $-3.87^{\mathrm{a} * * * \mathrm{~b} * * * *}$ & $36.27 \pm 0.22$ & $35.43 \pm 0.55$ & $-2.37^{\mathrm{a}^{* * * * 6 * * * *}}$ & $27.97 \pm 0.32$ & $27.02 \pm 0.18$ & $-3.52^{\mathrm{a}^{*} \mathrm{~b}^{* * * *}}$ \\
\hline $600 \mathrm{mg} / \mathrm{kg} \mathrm{BF}$ & $60.75 \pm 0.33$ & $59.20 \pm 0.26$ & $-2.62^{\mathrm{a}^{* * * * * * * * * *}}$ & $35.79 \pm 0.32$ & $35.13 \pm 0.39$ & $-1.88^{\mathrm{a}^{* * * * * * * * * *}}$ & $27.66 \pm 0.52$ & $26.88 \pm 0.23$ & $-2.90^{\mathrm{a} * * \mathrm{~b} * * *}$ \\
\hline $2 \%$ TW80 & $59.27 \pm 0.43$ & $54.05 \pm 0.63$ & -8.70 & $36.5 \pm 0.24$ & $34.59 \pm 0.44$ & -5.23 & $28.402 \pm 0.29$ & $27.072 \pm 0.33$ & -4.68 \\
\hline CQ25 mg/kg & $60.13 \pm 0.36$ & $59.86 \pm 0.23$ & $-0.45^{\mathrm{a}^{* * * *}}$ & $35.49 \pm 0.55$ & $35.48 \pm 0.67$ & $-0.03^{\mathrm{a}^{* * * *}}$ & $28.102 \pm 0.18$ & $28.422 \pm 0.22$ & $-1.14^{2 * * *}$ \\
\hline $200 \mathrm{mg} / \mathrm{kg} \mathrm{AF}$ & $59.58 \pm 0.55$ & $55.01 \pm 0.17$ & $-7.67^{\mathrm{a}^{* * * * * * * * * * * *}}$ & $36.38 \pm 0.08$ & $34.95 \pm 0.77$ & $-3.93^{\mathrm{a}^{*} \mathrm{~b}^{* * * * *}}$ & $28.172 \pm 0.35$ & $26.972 \pm 0.29$ & $-4.26^{\mathrm{b}^{* * *}}$ \\
\hline $400 \mathrm{mg} / \mathrm{kg} \mathrm{AF}$ & $60.05 \pm 0.73$ & $55.7 \pm 0.39$ & $-7.24^{\mathrm{a} * * * * * * * *}$ & $36.24 \pm 0.55$ & $35.04 \pm 0.23$ & $-3.3 \mathrm{a}^{* * * b^{* * * *}}$ & $28.012 \pm 0.36$ & $26.822 \pm 0.24$ & $-4.25^{b * * * *}$ \\
\hline $600 \mathrm{mg} / \mathrm{kg} \mathrm{AF}$ & $60.69 \pm 0.43$ & $56.52 \pm 0.53$ & $-6.87^{\mathrm{a}^{* * * * * * * * * * * * *}}$ & $35.83 \pm 0.78$ & $34.91 \pm 0.19$ & $-2.57^{\mathrm{a**} \mathrm{b}^{\mathrm{*} * * *}}$ & $28.232 \pm 0.56$ & $27.122 \pm 0.22$ & $-3.93^{b * * * *}$ \\
\hline
\end{tabular}

Notes: Data are expressed as mean \pm SEM; $\mathrm{n}=6$; ${ }^{\mathrm{a}}$ Compared with negative control; ${ }^{\mathrm{b}} \mathrm{To}$ CQ25 mg/kg; ${ }^{\mathrm{C}} \mathrm{To} 200 \mathrm{mg} / \mathrm{kg} ;{ }^{\mathrm{d}} \mathrm{To} 400 \mathrm{mg} / \mathrm{kg} ;{ }^{\mathrm{e}} \mathrm{To} 600 \mathrm{mg} / \mathrm{kg} ;{ }^{*} \mathrm{p}<0.05 ;{ }^{* *} \mathrm{p}<0.0 \mathrm{I} ;{ }^{* * * *} \mathrm{p}<0.00 \mathrm{I}$ Abbreviations: CF, chloroform fraction; BF, butanol fraction; $A F$, aqueous fraction; $2 \%$ TW80, $2 \%$ Tween 80 ; CQ, chloroquine; $D_{0}$, pre-treatment value on day 0 ; $D_{4}$, posttreatment value on day 4.

n-butanol fraction exhibited a statistically significant lowering effect in parasitemia level as well as improvement of mean survival time in a dose-dependent fashion $(\mathrm{p}<0.001)$ compared with their negative controls. Furthermore, a substantial dose-dependent effect was also depicted with the highest dose in comparison with the other two doses (Table 5).

Additionally, considerable $(\mathrm{p}<0.001)$ preventive effects were shown at all doses of n-butanol compared with the negative controls, in both PCV and rectal temperature decline. Besides, prevention of lowering of body weight was maximum at the highest dose $(\mathrm{p}<0.001)$ followed by middle dose $(p<0.01)$ and lowest dose $(p<0.05)$ when compared with the

Table 5 Parasitemia and Survival Time of Infected Mice Treated with n-Butanol Fraction of the Stem Bark of $F$. angolensis in Rane's Test

\begin{tabular}{|c|c|c|c|}
\hline Groups & $\%$ Parasitemia & \% Suppression & $\begin{array}{l}\text { Survival Time } \\
\text { (Days) }\end{array}$ \\
\hline $2 \%$ TW80 & $72.63 \pm 0.43$ & - & $7.23 \pm 0.53$ \\
\hline $\mathrm{CQ} 25 \mathrm{mg} / \mathrm{kg}$ & $0.00 \pm 0.00$ & $100.00^{\mathrm{a} * * *}$ & $28.00 \pm 0.00^{a x^{* 2 k}}$ \\
\hline $200 \mathrm{mg} / \mathrm{kg}$ & $41.52 \pm 0.42$ & 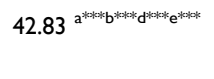 & 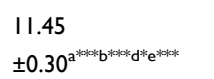 \\
\hline $400 \mathrm{mg} / \mathrm{kg}$ & $38.33 \pm 0.64$ & $47.23^{\mathrm{a}^{* * * *} \mathrm{~b}^{* * * *} \mathrm{e}^{* * * *}}$ & 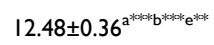 \\
\hline $600 \mathrm{mg} / \mathrm{kg}$ & $32.54 \pm 0.24$ & $54.20^{\mathrm{a}^{* * * *} \mathrm{~b}^{* * * *}}$ & 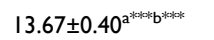 \\
\hline
\end{tabular}

Notes: Values are expressed as mean \pm SEM; $n=6$; ${ }^{a}$ Compared with negative control; ' ${ }^{\mathrm{T}} \mathrm{O}$ CQ25 mg/kg; ' $\mathrm{To} 200 \mathrm{mg} / \mathrm{kg}$; ${ }^{\mathrm{d}} \mathrm{To} 400 \mathrm{mg} / \mathrm{kg}$; ${ }^{\mathrm{e}} \mathrm{To} 600 \mathrm{mg} / \mathrm{kg}$; * ${ }^{*}<0.05$; $* * p<0.01 ; * * * p<0.001$.

Abbreviations: $2 \%$ TW80, $2 \%$ Tween 80 ; CQ, chloroquine. negative control groups (Table 6). In general, a dose-dependent effect was also noticed in all the three parameters i.e. PCV, rectal temperature, and body weight measurements.

\section{Antioxidant Activity of Crude Extract and Solvent Fractions}

The DPPH free radical scavenging activities of the crude extract and solvent fractions of stem bark of $F$. angolensis were concentration-dependent with an IC50 value of 2.72 $\pm 0.34,3.64 \pm 0.12,6.11 \pm 0.23,8.33 \pm 0.15$, and $14.93 \pm$ $0.44 \mathrm{mg} / \mathrm{mL}$ for the ascorbic acid, crude extract, n-butanol fraction, chloroform fraction and aqueous fraction, respectively. Higher free radical scavenging activity was observed in the n-butanol among other fractions. (Table 7)

\section{Preliminary Phytochemical Screening}

The phytochemical screening test revealed the presence of polyphenols, alkaloids, flavonoids, terpenoids, glycosides, and steroids, with an absence of saponins in the crude extract and all fractions. The aqueous fraction was also devoid of tannins (Table 8).

\section{Discussion}

In this experiment, the crude extract and solvent fractions of the plant stem bark were evaluated for antimalarial activities in terms of their schizontocidal activities during early infection in Peter's 
Table 6 Packed Cell Volume, Rectal Temperature and Body Weight of Infected Mice Treated with n-Butanol Fraction of the Stem Bark of $F$. angolensis in Rane's Test

\begin{tabular}{|c|c|c|c|c|c|c|c|c|c|}
\hline \multirow[t]{2}{*}{ Groups } & \multicolumn{3}{|c|}{ Packed Cell Volume } & \multicolumn{3}{|c|}{ Rectal Temperature } & \multicolumn{3}{|c|}{ Body Weight } \\
\hline & $\mathbf{D}_{3}$ & $\mathbf{D}_{7}$ & $\%$ Change & $\mathbf{D}_{3}$ & $\mathbf{D}_{7}$ & $\%$ Change & $\mathbf{D}_{3}$ & $\mathbf{D}_{7}$ & $\%$ Change \\
\hline $2 \%$ TW 80 & $50.96 \pm 0.65$ & $42.85 \pm 0.42$ & -18.93 & $35.23 \pm 0.24$ & $33.38 \pm 0.25$ & -5.54 & $27.99 \pm 0.45$ & $25.65 \pm 0.52$ & -9.12 \\
\hline $\mathrm{CQ} 25 \mathrm{mg} / \mathrm{kg}$ & $51.66 \pm 0.45$ & $52.34 \pm 0.25$ & $-1.30^{\mathrm{a} * * *}$ & $35.15 \pm 0.21$ & $35.35 \pm 0.19$ & $-0.57^{\mathrm{a}^{* * * * *}}$ & $28.49 \pm 0.52$ & $28.96 \pm 0.43$ & $-1.62^{\mathrm{a}^{* * * *}}$ \\
\hline 200 mg/kg & $51.08 \pm 0.28$ & $47.11 \pm 0.18$ & $-8.43^{\mathrm{a} * * * * * * * * * \mathrm{~d} * * \mathrm{e} * * * *}$ & $35.28 \pm 0.15$ & $33.88 \pm 0.20$ & $-4.13^{\mathrm{a} * * * \mathrm{~b} * * * *} \mathrm{~d} \cdot \mathrm{e}^{* * * *}$ & $27.86 \pm 0.33$ & $25.77 \pm 0.25$ & $-8.11^{\mathrm{a}^{*} \mathrm{~b}^{* * * *}}$ \\
\hline $400 \mathrm{mg} / \mathrm{kg}$ & $50.89 \pm 0.71$ & $48.16 \pm 0.22$ & $-5.67^{\mathrm{a} * * * \mathrm{~b}}$ & $35.26 \pm 0.35$ & $34.12 \pm 0.34$ & $-3.34^{\mathrm{a}^{* * * *} \mathrm{~b}^{* * * * k}}$ & $28.08 \pm 0.43$ & $26.12 \pm 0.42$ & $-7.50^{\mathrm{a} * * \mathrm{~b} * * * *}$ \\
\hline $600 \mathrm{mg} / \mathrm{kg}$ & $51.58 \pm 0.11$ & $49.36 \pm 0.17$ & $-4.50^{\mathrm{a} * * * b^{* * * * *}}$ & $35.23 \pm 0.19$ & $34.46 \pm 0.22$ & 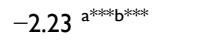 & $28.19 \pm 0.53$ & $26.35 \pm 0.32$ & $-6.98^{\mathrm{a}^{* * * *} \mathrm{~b}^{* * *}}$ \\
\hline
\end{tabular}

Notes: Values are expressed as mean \pm SEM; $n=6$; ${ }^{\mathrm{a} C o m p a r e d ~ w i t h ~ n e g a t i v e ~ c o n t r o l ; ~}{ }^{\mathrm{b}} \mathrm{To}$ CQ25 mg/kg; ${ }^{\mathrm{C}} \mathrm{To} 200 \mathrm{mg} / \mathrm{kg} ;{ }^{\mathrm{d}} \mathrm{To} 400 \mathrm{mg} / \mathrm{kg} ;{ }^{\mathrm{e}} \mathrm{To} 600 \mathrm{mg} / \mathrm{kg} ;{ }^{*} \mathrm{p}<0.05 ;{ }^{* *} \mathrm{p}<0.0 \mathrm{l}$; $* * * \mathrm{p}<0.001$.

Abbreviations: $2 \%$ TW80, $2 \%$ Tween80; CQ, chloroquine; $D_{3}$, pre-treatment value on day 3; $D_{7}$, post-treatment value on day 7 .

Table 7 Antioxidant Activities of the Crude Extract and Solvent Fractions of Stem Bark of $F$. angolensis in DPPH Assay Model

\begin{tabular}{|l|l|l|l|l|l|}
\hline \multicolumn{2}{|l|}{ Inhibition of DPPH } \\
\hline Concentration (mg/mL) & Aqueous Fraction & Chloroform Fraction & n- Butanol Fraction & Crude Extract & Ascorbic Acid \\
\hline 50 & $13.24 \pm 1.52$ & $30.07 \pm 0.80$ & $39.19 \pm 0.55$ & $44.37 \pm 0.66$ & $48.67 \pm 1.23$ \\
100 & $28.64 \pm 0.45$ & $43.54 \pm 1.11$ & $56.62 \pm 1.20$ & $65.40 \pm 0.57$ & $69.53 \pm 0.88$ \\
200 & $42.37 \pm 0.48$ & $55.22 \pm 0.12$ & $68.51 \pm 0.49$ & $72.37 \pm 1.02$ & $76.19 \pm 0.36$ \\
400 & $48.94 \pm 0.78$ & $65.37 \pm 0.75$ & $75.27 \pm 0.46$ & $78.79 \pm 0.66$ & $83.55 \pm 0.73$ \\
600 & $59.37 \pm 1.03$ & $70.79 \pm 0.34$ & $79.27 \pm 0.67$ & $82.48 \pm 0.59$ & $90.34 \pm 0.84$ \\
800 & $68.84 \pm 0.66$ & $78.64 \pm 0.47$ & $81.27 \pm 0.82$ & $85.49 \pm 0.64$ & $94.27 \pm 0.54$ \\
1000 & $71.42 \pm 0.26$ & $83.71 \pm 1.22$ & $86.44 \pm 1.34$ & $90.57 \pm 0.65$ & $98.07 \pm 0.18$ \\
$I C 50 \mathrm{mg} / \mathrm{mL}$ & $14.93 \pm 0.44$ & $8.33 \pm 0.15$ & $6.11 \pm 0.23$ & $3.64 \pm 0.12$ & $2.72 \pm 0.34$ \\
\hline
\end{tabular}

Notes: The values are presented as means + standard error of the mean (SEM); $n=3$, triplicate measurements.

Abbreviation: IC50, 50\% inhibitory concentration.

Table 8 Preliminary Phytochemical Screening of $80 \%$ Methanol Extract and Solvent Fractions of Stem Bark of F. angolensis

\begin{tabular}{|l|l|l|l|l|l|}
\hline $\begin{array}{l}\text { Secondary } \\
\text { Metabolites }\end{array}$ & $\begin{array}{l}\text { Reagents/ } \\
\text { Chemicals }\end{array}$ & $\begin{array}{l}\text { Crude } \\
\text { Extract }\end{array}$ & $\begin{array}{l}\text { Chloroform } \\
\text { Fraction }\end{array}$ & $\begin{array}{l}\text { n-Butanol } \\
\text { Fractions }\end{array}$ & $\begin{array}{l}\text { Aqueous } \\
\text { Fraction }\end{array}$ \\
\hline Alkaloid & Mayer's reagent & + & + & + & + \\
Flavonoids & Lead acetate & + & + & + & + \\
Phenols & I\% FeCl3 & + & + & + & + \\
Terpenoids & H2SO4\& chloroform & + & + & - & + \\
Saponins & Vigorous shaking & - & - & + & - \\
Tannins & $0.1 \%$ ferric chloride & + & + & + & + \\
Steroids & H2SO4\& chloroform & + & + & + & + \\
Glycosides & Glacial acetic acid & + & + & & + \\
\hline
\end{tabular}

Notes: ${ }^{+}$Presence; ${ }^{-}$Absence.

4-day suppressive test, and the curative potential of the most active fraction during established infection in the Rane's test.

In the present study, the acute oral toxicity study revealed that the plant neither produced gross behavioral changes nor mortality in mice indicating that the median lethal dose was beyond $2000 \mathrm{mg} / \mathrm{kg}$, according to OECD guideline no. 425 and thus, the safety of the plant in the folklore antimalarial use seems to be reliable.
According to this study, the crude extract and fractions of the plant produced promising antioxidant activity. Among fractions, higher free radical scavenging activity (86.44\%) was observed in the n-butanol while the lowest effect $(71.42 \%)$ was observed in the aqueous fraction. The percentage of inhibition of the crude extract and ascorbic acid was found to be $90.57 \%$ and $98.07 \%$, respectively. 
In a 4-day suppressive test, the percent of parasitemia suppression and mean survival time were determined and compounds were assumed effective when parasitemia suppression exceeded $30 \%{ }^{42}$ In this study, the dose-dependent inhibition of parasitemia and prolongation of survival time were observed at all the doses of the crude extract. Therefore, it is safe to assume that the plant could produce considerable schizontocidal activity, so that the primary attack due to an early infection can be mitigated ${ }^{34}$ and the overall pathogenic effect of the parasite was substantially reduced. $^{43}$

The maximum chemo-suppressive effect was seen in the n-butanol fraction followed by chloroform and aqueous fraction with a percentage parasitemia suppression of $52.93,40.5$, and 19.55 respectively at their highest doses. Likewise, survival time improvement was highest in the nbutanol fraction followed by the chloroform fraction. This shows that the type and content of bioactive secondary metabolites responsible for antimalarial activity are more concentrated in n-butanol and chloroform fractions. The higher chemo-suppressive effect of n-butanol fraction compared with the other two fractions could imply that active parts are concentrated in relatively non-polar and semi-polar solvents. ${ }^{33,44}$ However, the fractions had lower effects of parasitemia suppression as well as MST than the crude extract, suggesting that fractionation might lead to loss of additive or synergistic activity and less concentration of secondary metabolites, as evidenced by other studies. ${ }^{6,45}$

The curative potential of the n-butanol fraction was further investigated in Rane's test. In this study, the nbutanol fraction exhibited substantial inhibition of parasitemia and improved survival time. Over the course of treatment, a significant difference $(\mathrm{p}<0.001)$ in parasite development was shown upon the two-way repeated measures ANOVA analysis of parasitemia. Moreover, significant parasitemia suppression of the fraction was commenced after the initial dose, compared with the negative control (Figure 1). This indicates that the plant had profound rapid and sustained antimalarial effects in early and late infections in a dose-dependent manner, supported by similar studies ${ }^{7,46}$ and supports the in vitro antiplasmodial activity of $F$. angolensis as well as its ethnobotanical uses. $^{12-14}$

On the other hand, prevention of body weight loss, amelioration of anemia, and stabilization of temperature in infected mice are important actions of a potent antimalarial drug. ${ }^{6,47}$ In this regard, the plant had a promising effect on these parameters as revealed by this study. Protective effects of the crude extract against a drop in PCV and rectal temperature were statistically significant $(p<0.001)$ in all the three doses compared with their respective negative controls (Table 2). This finding could infer that the plant has good protective effects against

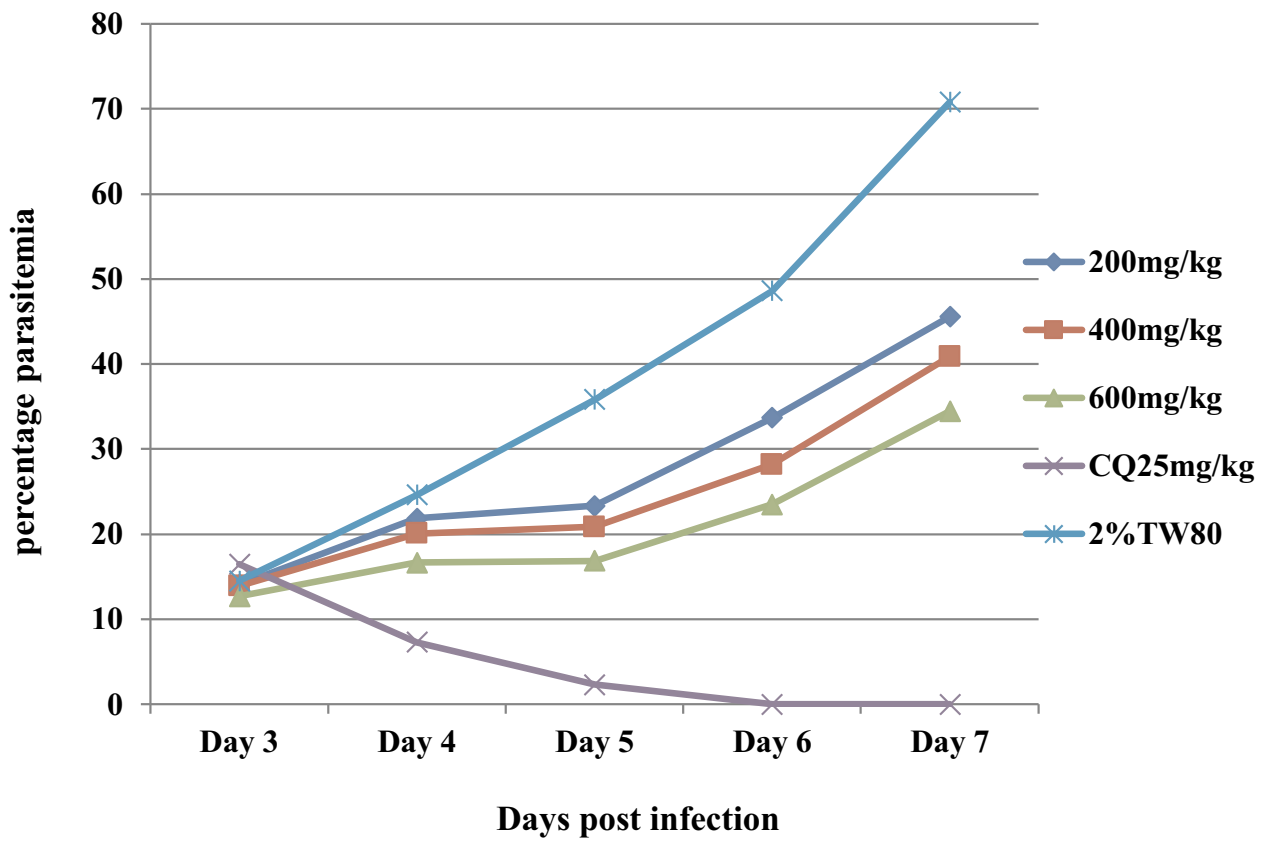

Figure I Parasitemia development over the course of treatment with n-butanol fraction of the stem bark of $F$. angolensis in Rane's model. 
hemolysis of parasitized erythrocytes and considerable effect towards controlling some pathological processes and the immune system and thus, preventing a further drop in metabolic rates and body temperature. Previous reports indicated that increased parasitemia is associated with decreased metabolic rates and reduced temperature that may lead to death. ${ }^{48}$ Furthermore, malaria infection is accompanied by bodyweight reduction as a result of decreased food intake, disturbed metabolic function, and hypoglycemia. ${ }^{6,43}$ The crude extract produced a moderate effect in ameliorating a decrease in body weight. This effect correlates with the overall improvement in PCV, body temperature, and parasitic clearance. ${ }^{7}$ Moreover, the antioxidant effect of the crude extract has a substantial contribution to prevent PCV reduction resulting from oxidative stress-associated hemolysis of RBCs, as supported by previous reports. ${ }^{49,50}$

Likewise, in the 4-day suppressive test, all fractions at the three doses produced statistically significant protective effects $(\mathrm{p}<0.001)$ in PCV and rectal temperature decline when compared with the negative controls. However, the n-butanol fraction being the most active fraction had the highest circumvention effect $(p<0.001)$ in PCV and rectal temperature reduction in both models. The maximum antioxidant activity alongside the bioactive constituents either individually or synergistically may be responsible for its profound antimalarial activity in both models.

The current phytochemical screening revealed that both the crude extract and the fractions are endowed with different secondary metabolites notably polyphenols, flavonoids, terpenoids, steroids, alkaloids, and glycosides. Interestingly, from the stem bark of $F$. angolensis, it was possible to isolate 6-acetonyl derivatives of the benzophenanthridine alkaloids such as dihydrochelerythrine, dihydronitidine, and dihydrosanguinarine that promise to have antioxidant and antimalarial activities. ${ }^{51}$ This finding was further supported by similar studies which have indicated that secondary plant metabolites such as alkaloids, terpenes, flavonoids, xanthones, anthraquinones, phenolic compounds, sesquiterpenes, and other related compounds have shown antimalarial activities. For instance, sesquiterpenes (such as artemisinin) and alkaloids (such as chloroquine) exert their antimalarial effect by the formation of potentially toxic heme-adducts, among many other compounds. ${ }^{52,53}$ Therefore, the study recommends further isolation, characterization, structural elucidation and the study of the structure-activity relationship of the active constituents responsible for this activity.
Generally, the present study together with the previous in vitro antiplasmodial activity could justify the traditional uses of the plant in the treatment of malaria.

\section{Conclusion}

This study concluded that the plant had promising antimalarial activity as evidenced by the significant reduction in parasitemia level, improvement of mean survival time, and substantial protective effects in the other surrogate parameters in a dose-dependent manner. Among all fractions, the n-butanol fraction exhibited maximum antimalarial activity in both models. The secondary metabolites and good antioxidant activity of $F$. angolensis together may contribute to its antiplasmodial activity.

\section{Abbreviations}

DPPH, diphenyl-2-picrylhydrazyl; OECD, Organization for Economic Cooperation and Development; PCV, packed cell volume; RBC, red blood cell; SEM, standard error of the mean; WHO, World Health Organization.

\section{Data Sharing Statement}

All data generated or analyzed during this study are included in the manuscript and are also available from the corresponding author upon request.

\section{Ethics Approval}

Ethical approval was obtained from the Research and Ethical Committee of the Department of Pharmacy, Wollo University. All the experiments were conducted in accordance with a guide for the care and use of laboratory animals: Eighth Edition by National Research Council, Division on Earth and Life Studies, Institute for Laboratory Animal Research, Committee for the Update of the Guide for the Care and Use of Laboratory Animals. We also followed OECD guideline no. 425. Then, the animals were killed following the standard protocol at the end of the experiment.

\section{Acknowledgment}

We greatly thank Wollo University for use of the laboratory to conduct the experiment and its financial support.

\section{Author Contributions}

All authors made substantial contributions to the conception and design, acquisition of data, or analysis and interpretation of data; took part in drafting the article or revising it critically for important intellectual content; 
agreed to submit to the current journal; gave final approval of the version to be published; and agree to be accountable for all aspects of the work.

\section{Funding}

Wollo University covered the costs of chemicals, reagents and equipments used.

\section{Disclosure}

The authors declare that they have no potential conflicts of interest.

\section{References}

1. Srivastava A, Creek DJ, Evans KJ, et al. Host reticulocytes provide metabolic reservoirs that can be exploited by malaria parasites. PLoS Pathog. 2015;11(6):e1004882. doi:10.1371/journal.ppat.1004882

2. Hanboonkunupakarn B, White NJ. The threat of antimalarial drug resistance. Trop Dis Travel Med Vaccines. 2016;2(1):10. doi:10.1186/ s40794-016-0027-8

3. World Health Organization. World malaria report 2016. Geneva: World Health Organization; 2016.

4. Dvorin JD. Getting your head around cerebral malaria. Cell Host Microbe. 2017;22(5):586-588. doi:10.1016/j.chom.2017.10.017

5. Mutegeki E, Chimbari MJ, Mukaratirwa S. Assessment of individual and household malaria risk factors among women in a South African village. Acta Trop. 2017;175:71-77.

6. Misganaw D, Engidawork E, Nedi T. Evaluation of the anti-malarial activity of crude extract and solvent fractions of the leaves of Olea europaea (Oleaceae) in mice. BMC Complement Altern Med. 2019;19 (1):171. doi:10.1186/s12906-019-2567-8

7. Misganaw D, Amare GG, Mengistu G. Chemo suppressive and curative potential of hypoestes forskalei against Plasmodium berghei: evidence for in vivo antimalarial activity. $J$ Exp Pharmacol. 2020;12:313. doi:10.2147/JEP.S262026

8. Shelton JM, Corran P, Risley P, et al. Genetic determinants of antimalarial acquired immunity in a large multi-centre study. Malar $J$. 2015;14(1):333. doi:10.1186/s12936-015-0833-x

9. Karunamoorthi K, Girmay A, Fekadu S. Larvicidal efficacy of Ethiopian ethnomedicinal plant Juniperus procera essential oil against Afrotropical malaria vector Anopheles arabiensis (Diptera: culicidae). Asian Pac J Trop Biomed. 2014;4:S99-S106. doi:10.12980/ APJTB.4.2014C687

10. B DM LN A, Bharucha B, Dwivedi M, Laddha NC. Antioxidant rich flavonoids from Oreocnide integrifolia enhance glucose uptake and insulin secretion and protects pancreatic $\beta$-cells from streptozotocin insult. BMC Complement Altern Med. 2011;11(1):126. doi:10.1186/ 1472-6882-11-126

11. Pascaline J, Charles M, George O, Lukhoba C. An inventory of medicinal plants that the people of Nandi use to treat malaria. $J$ Anim Plant Sci. 2011;9:1192-1200.

12. Ruth L, Manani Solomon D. Ethnobotanical survey and propagation of some endangered medicinal plants from south Nandi district of Kenya. J Anim Plant Sci. 2010;8(3):1016-1043.

13. Kareru P, Kenji G, Gachanja A, Keriko J, Mungai G. Traditional medicines among the Embu and Mbeere people of Kenya. Afr $J$ Tradit Complement Altern Med. 2007;4(1):75-86. doi:10.4314/ajtcam.v4i1.31193

14. Asnake S, Teklehaymanot T, Hymete A, Erko B, Giday M. Survey of medicinal plants used to treat malaria by Sidama People of Boricha District, Sidama Zone, South Region of Ethiopia. Evid Based Complement Alternat Med. 2016;2016:1-9. doi:10.1155/2016/9690164
15. Nguta J, Mbaria J, Gakuya D, Gathumbi P, Kiama S. Antimalarial herbal remedies of Msambweni, Kenya. J Ethnopharmacol. 2010;128 (2):424-432. doi:10.1016/j.jep.2010.01.033

16. Shiracko N, Owuor BO, Gakuubi MM, Wanzala W. A survey of ethnobotany of the AbaWanga people in Kakamega County, western province of Kenya. 2016.

17. Eshete MA, Kelbessa E, Dalle G. Ethnobotanical study of medicinal plants in Guji agro-pastoralists, Blue Hora District of Borana Zone, Oromia region, Ethiopia. J Med Plants Stud. 2016;4(2):170184.

18. Fenetahun Y, Eshetu G. A review on ethnobotanical studies of medicinal plants use by agro-pastoral communities in, Ethiopia. $J$ Med Plants. 2017;5(1):33-44.

19. Gradé JT, Tabuti JR, Van Damme P. Ethnoveterinary knowledge in pastoral Karamoja, Uganda. J Ethnopharmacol. 2009;122(2):273293. doi:10.1016/j.jep.2009.01.005

20. Mothana RA, Al-Musayeib NM, Al-Ajmi MF, Cos P, Maes L. Evaluation of the in vitro antiplasmodial, antileishmanial, and antitrypanosomal activity of medicinal plants used in Saudi and Yemeni traditional medicine. Evid Based Complement Alternat Med. 2014;2014. doi:10.1155/2014/905639

21. Muthaura C, Keriko J, Mutai C, et al. Antiplasmodial potential of traditional phytotherapy of some remedies used in treatment of malaria in Meru-Tharaka Nithi County of Kenya. $J$ Ethnopharmacol. 2015;175:315-323. doi:10.1016/j.jep.2015.09.017

22. Al Haidari RA. A review of traditional uses, phytochemicals and bioactivities of the Genus hypoestes. Afr J Tradit Complement Altern Med. 2018;15(3):1-17.

23. Kuglerova M, Tesarova H, Grade JT, et al. Antimicrobial and antioxidative effects of Ugandan medicinal barks. Afr J Biotechnol. 2011;10(18):3628-3632.

24. Yiaile AL, Mbaria JM, Ole-Mapenay IM, Okumu MO, Hadun AH, Onyancha JM. Preliminary screening of crude extracts of fagaropsis angolensis for anticancer activity. 2018.

25. Lacroix D, Prado S, Kamoga D, et al. Antiplasmodial and cytotoxic activities of medicinal plants traditionally used in the village of Kiohima, Uganda. $J$ Ethnopharmacol. 2011;133(2):850-855. doi:10.1016/j.jep.2010.11.013

26. Habinshuti J, Akenga T. In vitro anti-malarial activity evaluation of extracts from plants often used in the East African Region. Eur J Med Plants. 2018;22:1-11. doi:10.9734/EJMP/2018/40227

27. Kirira P, Rukunga G, Wanyonyi A, et al. Anti-plasmodial activity and toxicity of extracts of plants used in traditional malaria therapy in Meru and Kilifi Districts of Kenya. J Ethnopharmacol. 2006;106 (3):403-407. doi:10.1016/j.jep.2006.01.017

28. Asrade S, Mengesha Y, Moges G, Gelayee DA. In vivo antiplasmodial activity evaluation of the leaves of Balanites rotundifolia (Van Tiegh) blatter (Balanitaceae) against Plasmodium berghei. J Exp Pharmacol. 2017;9:59.

29. Organization for Economic Cooperation and Development. OECD Guideline for the testing of chemicals: acute oral toxicity; up-anddown procedure (UDP). OECD, No 425. 2008.

30. Guideline P-BT. OECD guideline for the testing of chemicals. Hershberger. 2001;601:858.

31. Gurmu AE, Birru EM, Geta M. Antiplasmodial activity of Indigofera spicata root extract against Plasmodium berghei infection in mice. Malar J. 2017;16(1):198. doi:10.1186/s12936-0171853-5

32. Fidock DA, Rosenthal PJ, Croft SL, Brun R, Nwaka S. Antimalarial drug discovery: efficacy models for compound screening. Nat Rev Drug Discov. 2004;3(6):509-520. doi:10.1038/nrd1416

33. Bantie L, Assefa S, Teklehaimanot T, Engidawork E. In vivo antimalarial activity of the crude leaf extract and solvent fractions of Croton macrostachyus Hocsht. (Euphorbiaceae) against Plasmodium berghei in mice. BMC Complement Altern Med. 2014;14:79. doi:10.1186/1472-6882-14-79 
34. Peters W, Portus J, Robinson B. The four-day suppressive in vivo antimalarial test. Ann Trop Med Parasitol. 1975;69:155-171. doi:10. 1080/00034983.1975.11686997

35. Ryley J, Peters W. The antimalarial activity of some quinolone esters. Ann Trop Med Parasitol. 1970;64(2):209-222. doi:10.1080/000349 83.1970.11686683

36. Peters W, Robinson B. The chemotherapy of rodent malaria. XLVII. Studies on pyronaridine and other Mannich base antimalarials. Ann Trop Med Parasitol. 1992;86(5):455-465. doi:10.1080/00034983. 1992.11812694

37. Mekonnen LB. In vivo antimalarial activity of the crude root and fruit extracts of Croton macrostachyus (Euphorbiaceae) against Plasmodium berghei in mice. J Tradit Complement Med. 2015;5 (3):168-173. doi:10.1016/j.jtcme.2014.07.002

38. Trease G, Evans M. Text Book of Pharmacognosy. 13th ed. London, Toronto: Bailiere Tindall, Tokyo Pgs; 1989:200-201.

39. Debella A. Manual for phytochemical screening of medicinal plants, Department of Drug Research, Ethiopian Nutrition and Research Institute. Addis Ababa, Ethiopia; 2002:45-71.

40. Blois MS. Antioxidant determinations by the use of a stable free radical. Nature. 1958;181(4617):1199-1200. doi:10.1038/1811199a0

41. Desmarchelier C, Novoa Bermudez M, Coussio J, Ciccia G, Boveris A. Antioxidant and prooxidant activities in aqueous extracts of Argentine plants. Int J Pharmacogn. 1997;35(2):116-120. doi:10.10 76/phbi.35.2.116.13282

42. Krettli AU, Adebayo JO, Krettli LG. Testing of natural products and synthetic molecules aiming at new antimalarials. Curr Drug Targets. 2009;10(3):261-270. doi:10.2174/138945009787581203

43. Basir R, Rahiman SF, Hasballah K, et al. Plasmodium berghei ANKA infection in ICR mice as a model of cerebral malaria. Iran $J$ Parasitol. 2012;7(4):62.

44. Taherkhani M, Rustaiyan A, Nahrevanian H, Naeimi S, Taherkhani T. Comparison of antimalarial activity of Artemisia turanica extract with current drugs in vivo. $J$ Vector Borne Dis. 2013;50(1):51.
45. Traore M. Investigation of antiplasmodial compounds from two plants, Cochlospermum tinctorium a rich and Gardenia sokotensis hutch. Afr J Tradit Complement Altern Med. 2006;3(4):34-41. doi:10.4314/ajtcam.v3i4.31175

46. Olorunniyi O. In vivo antimalarial activity of crude aqueous bark extract of Trichilia monadelpha against Plasmodium berghei berghei (NK65) in mice. Int J Pharm Med Bio Sci. 2013;2(4):2278-5221.

47. Böttger S, Melzig MF. The influence of saponins on cell membrane cholesterol. Bioorg Med Chem. 2013;21(22):7118-7124. doi:10.10 16/j.bmc.2013.09.008

48. Felter H, Lloyd J. King's American Dispensatory. 3rd revision ed. Cincinnati, OH: Ohio Valley Co; 1898.

49. Kremsner P, Greve B, Lell B, Luckner D, Schmid D. Malarial anaemia in African children associated with high oxygen-radical production. Lancet. 2000;355(9197):40-41. doi:10.1016/S0140-6736 (99)04761-3

50. Loria P, Miller S, Foley M, Tilley L. Inhibition of the peroxidative degradation of haem as the basis of action of chloroquine and other quinoline antimalarials. Biochem J. 1999;339(2):363-370. doi:10.10 42/bj3390363

51. Khalid SA, Waterman PG. 6-Hydroxymethyldihydronitidine from Fagaropsis angolensis. J Nat Prod. 1985;48(1):118-119. doi:10.10 21/np50037a022

52. Soares JBC, Menezes D, Vannier-Santos MA, et al. Interference with hemozoin formation represents an important mechanism of schistosomicidal action of antimalarial quinoline methanols. PLoS Negl Trop Dis. 2009;3(7):e477. doi:10.1371/journal.pntd.0000477

53. Eastman RT, Fidock DA. Artemisinin-based combination therapies: a vital tool in efforts to eliminate malaria. Nat Rev Microbiol. 2009;7 (12):864-874. doi:10.1038/nrmicro2239

\section{Publish your work in this journal}

The Journal of Experimental Pharmacology is an international, peerreviewed, open access journal publishing original research, reports, reviews and commentaries on all areas of laboratory and experimental pharmacology. The manuscript management system is completely online and includes a very quick and fair peer-review system. Visit http://www.dovepress.com/testimonials.php to read real quotes from published authors. 